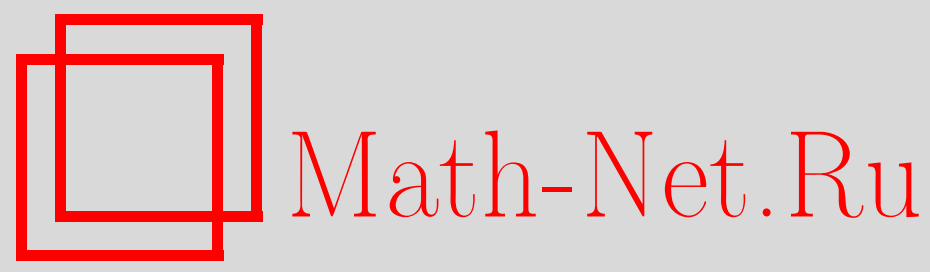

С. П. Горбиков, Локальные особенности динамических систем с ударными взаимодействиями, Матем. заметки, 1998, том 64, выпуск 4, 531-542

DOI: https://doi.org/10.4213/mzm1427

Использование Общероссийского математического портала Math-Net.Ru подразумевает, что вы прочитали и согласны с пользовательским соглашением http://www.mathnet.ru/rus/agreement

Параметры загрузки:

IP: 54.162 .85 .209

26 апреля 2023 г., $13: 15: 36$

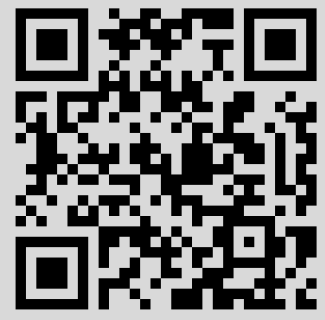




\title{
ЛОКАЛЬНЫЕ ОСОБЕННОСТИ ДИНАМИЧЕСКИХ СИСТЕМ С УДАРНЫМИ ВЗАИМОДЕЙСТВИЯМИ
}

\author{
С. П. Горбиков
}

\begin{abstract}
Для выделенного класса динамических систем с ударными взаимодействиями проведена классификация локальных (качественных) особенностей. Для первых четырех выделенных типов локальных особенностей описаны такие свойства качественной структуры, с помощью которых затем устанавливается топологическая эквивалентность соответствующих особенностей.

Библиографоия: 11 названий.
\end{abstract}

Введение. Изучение динамических систем с ударными взаимодействиями проводилось давно и во многих работах, однако построение качественной теории таких систем происходило разрозненно, несмотря на то, что качественная теория гладких динамических систем к настоящему времени довольно хорошо развита ([1]-[3] и др.) и для негладких динамических систем определенного вида качественная теория успешно развивается ([4] и др.).

Для динамических систем с ударными взаимодействиями при изучении локальных особенностей: в [5] для конкретной системы, а в [6] для неавтономной системы общего вида - описываются некоторые особенности качественной структуры фазового пространства в малой окрестности такой точки на поверхности $S=0$ удара, в которой первая и вторая производные (в силу дифференциальных уравнений движения) уравнения этой поверхности равны 0, а третья производная положительна (движение системы происходит в области $S \geqslant 0$ ). В [7], [8] для динамических систем с ударньми взаимодействиями общего вида изучается структура фазового пространства малых окрестностей точек, лежащих на многообразии удара.

В данной работе для выделенного класса динамических систем с ударньпи взаимодействиями классифицируются их локальные особенности. Для первых четырех выделенных типов локальных особенностей описаны такие свойства качественной структуры, с помощью которьх затем устанавливается топологическая эквивалентность соответствующих особенностей. Тем самьм, дается полное в смысле Андронова-Понтрягина описание указанных локальных особенностей.

1. Уравнения движения. Рассматривается следующий обший вид [8] динамической системы с ударньми взаимодействиями. Мгновенное ударное взаимодействие происходит на гиперповерхности $x_{n}=0$, по достижении которой фазовые переменные $x_{1}, x_{2}, \ldots, x_{n-1}$ меняются скачкообразно (переменная $x_{n}$ остается равной 0 ) согласно 
формулам

$$
\begin{gathered}
\bar{x}_{1}=H_{1}\left(x_{1}^{-}, \ldots, x_{n-1}^{-}\right)=x_{1}^{-} H_{11}\left(x_{1}^{-}, \ldots, x_{n-1}^{-}\right), \\
\bar{x}_{i}=H_{i}\left(x_{1}^{-}, \ldots, x_{n-1}^{-}\right)=x_{i}^{-}+x_{1}^{-} H_{1 i}\left(x_{1}^{-}, \ldots, x_{n-1}^{-}\right), \quad i=2, \ldots, n-1,
\end{gathered}
$$

а при $x_{n}>0$ изменение фазовых переменных подчиняется дифференциальньм уравнениям вида

$$
\begin{gathered}
\dot{x}_{i}=\Phi_{i}\left(x_{1}, \ldots, x_{n}\right), \quad i=1, \ldots, n-1, \\
\dot{x}_{n}=\Phi_{n}\left(x_{1}, \ldots, x_{n}\right)=x_{1} \Phi_{n 1}\left(x_{1}, \ldots, x_{n}\right)+x_{n} \Phi_{n n}\left(x_{1}, \ldots, x_{n}\right) .
\end{gathered}
$$

Фазовое пространство системы составляют точки $\left(x_{1}, \ldots, x_{n-1}, x_{n} \geqslant 0\right)$. В соотношениях (1) и $(2)\left(x_{1}^{-}, \ldots, x_{n-1}^{-}, 0\right)$ и $\left(\bar{x}_{1}, \ldots, \bar{x}_{n-1}, 0\right)-$ соответственно доударные и послеударные значения переменных;

$$
\begin{gathered}
-1 \leqslant H_{11}\left(0, x_{2}^{-}, \ldots, x_{n-1}^{-}\right) \leqslant 0, \quad H_{11}\left(x_{1}^{-}, x_{2}^{-}, \ldots, x_{n-1}^{-}\right) \leqslant 0 \\
\Phi_{n 1}\left(x_{1}, \ldots, x_{n-1}, 0\right)>0 .
\end{gathered}
$$

Кроме того, функции $H_{1 j}$ и $\Phi_{j}, j=1, \ldots, n-1, \Phi_{n 1}, \Phi_{n n}$ определены и являются гладкими класса $C^{m}, m \geqslant 3$, в мальх окрестностях точек $\left(x_{1}^{-} \leqslant 0, x_{2}^{-}, \ldots, x_{n-1}^{-}\right)$и $\left(x_{1}, \ldots, x_{n-1}, x_{n} \geqslant 0\right)$ соответственно.

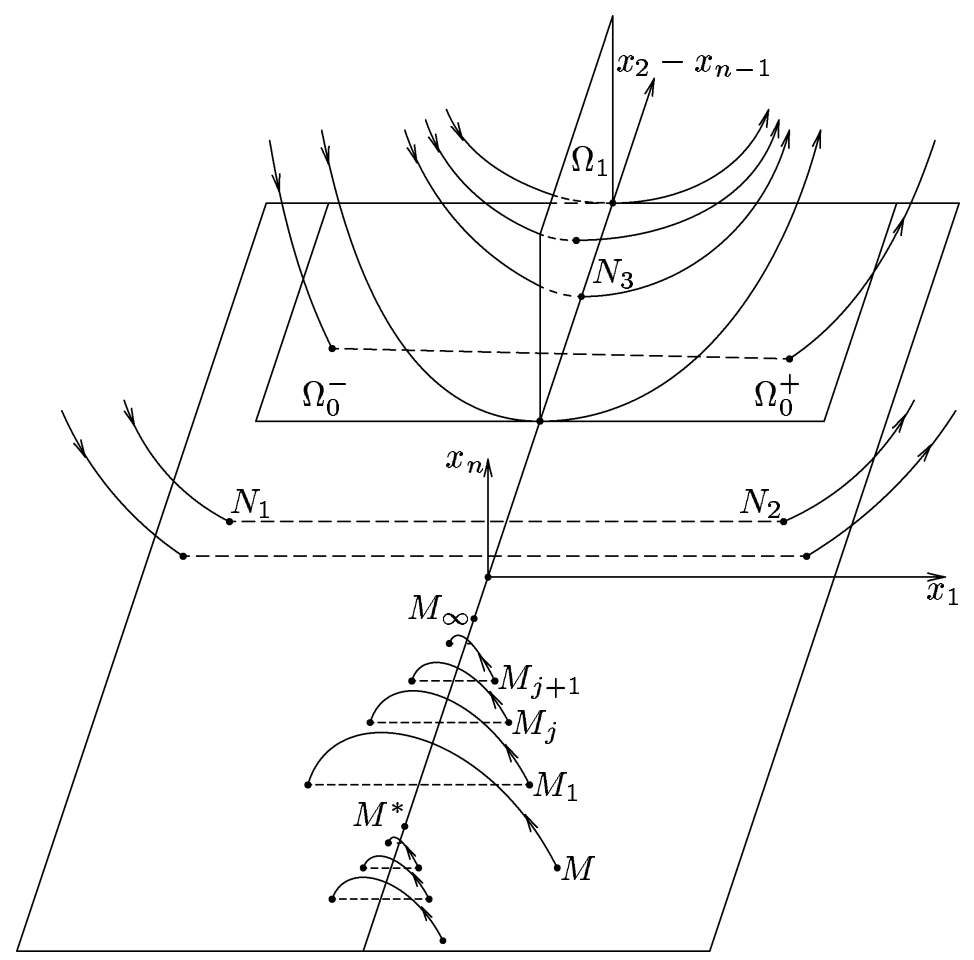

Рис. 1 
Специфический вид уравнений (2) и условие (3) означают лишь, что

1) на гиперповерхности $x_{n}=0$ согласно $(2)$

$$
\dot{x}_{n}=x_{1} \Phi_{n 1}\left(x_{1}, \ldots, x_{n-1}, 0\right) ;
$$

$2)$ в силу $\left[9\right.$, с. 171] фазовые траектории системы (2) при $x_{1}=0$ касаются гиперповерхности $x_{n}=0$, при возрастании времени $t$ они выходят в область $x_{n}>0$ из точек $\left(x_{1}>0, x_{2}, \ldots, x_{n-1}, x_{n}=0\right)$, а при уменьшении $t-$ из точек $\left(x_{1}<0, x_{2}\right.$, $\left.\ldots, x_{n-1}, 0\right)$

(на рис. 1 сплошными линиями обозначены траектории системы (2), пунктирными линиями соединены точки и их образы при отображении (1)).

2. Классификация локальных особенностей. Далее изучается топологическая (качественная) структура динамической системы (1), (2) в малых окрестностях точек $M^{*}$ в предположении, что $M^{*}$ принадлежит гиперповерхности $x_{n}=0$ (достаточно полное описание случая $M^{*}=\left(x_{1}^{*}, \ldots, x_{n-1}^{*}, x_{n}^{*}>0\right)$ известно из качественной теории гладких дифференциальных уравнений).

Кроме того, предполагается, что в точках многообразия $x_{n}=0$ все функции $\Phi_{i}\left(x_{1}\right.$, $\left.x_{2}, \ldots, x_{n}\right), i=1, \ldots, n$, не могут одновременно обращаться в 0 .

Из определений, принятых в качественной теории дифференциальных уравнений (см. [1]-[4]), видно, что все точки $M^{*}$ многообразия $x_{n}=0$ являются топологически особыми (локальными особенностями). Будем различать следующие типы локальных особенностей $M^{*}$ динамической системы $(1),(2)$ :

1) в точке $M^{*}$ выполняются условия

$$
x_{n}=0, \quad \dot{x}_{n}=\Phi_{n}\left(x_{1}, \ldots, x_{n}\right)<0 ;
$$

2) в точке $M^{*}$ выполняются условия

$$
x_{n}=0, \quad \dot{x}_{n}>0 ;
$$

3) в точке $M^{*}$ вьполняются условия

$$
x_{n}=0, \quad \dot{x}_{n}=0, \quad \ddot{x}_{n}=\sum_{k=1}^{n} \frac{\partial \Phi_{n}}{\partial x_{k}} \Phi_{k}>0 ;
$$

4) в точке $M^{*}$ вьполняются условия

$$
x_{n}=0, \quad \dot{x}_{n}=0, \quad \ddot{x}_{n}<0 ;
$$

5) в точке $M^{*}$ вьполняются условия

$$
x_{n}=0, \quad \dot{x}_{n}=0, \quad \ddot{x}_{n}=0, \quad \dddot{x}_{n}=\sum_{j=1}^{n} \frac{\partial}{\partial x_{j}}\left(\sum_{k=1}^{n} \frac{\partial \Phi_{n}}{\partial x_{k}} \Phi_{k}\right) \Phi_{j}>0 ;
$$

6) в точке $M^{*}$ выполняются условия

$$
x_{n}=0, \quad \dot{x}_{n}=0, \quad \ddot{x}_{n}=0, \quad \dddot{x}_{n}<0 .
$$

Типы 5), 6) локальных особенностей изучались ранее [5], [6], [8], однако исследование условий их топологической эквивалентности требует отдельного рассмотрения и здесь не проводится.

Более вырожденные случаи (когда в точке $M^{*}$ первой отличной от 0 производной функции $x_{n}$ является четвертая или еще более высокая) здесь также не рассматриваются. 


\section{3. Изучение локальных особенностей типов 1)-3).}

Лемма 1. Пусть в точке $M^{*}$ выполняются условия (5). Тогда существует такая достаточно малая окрестность $\Omega$ этой точки в фазовом пространстве системы (1), (2), через любую точку которой при $x_{n}>0$ проходит фазовая траектория, приводящая за достаточно мальй промежуток времени фазовую точку на многообразие удара $x_{n}=0$. В силу действующих затем ударных взаимодействий фазовая точка покидает выбранную малую окрестность $\Omega$.

Для любых систем вида (1), (2) (при одном и том же значении n) локальные особенности вида (5) имеют малые окрестности в фазовом пространстве соответствующих систем, топологическая структура которых одинакова.

На рис. 1 особенности такого типа соответствует, например, точка $N_{1}$.

ДокАЗАтЕЛьСтво. На гиперповерхности удара $x_{n}=0$ можно выбрать такую достаточно малую окрестность $\Omega_{0}\left(M^{*}\right)$ точки $M^{*}$, во всех точках которой вьполняются условия (5), а значит, и неравенство $x_{1}<0$ (в силу (4) и (3)). Поэтому

а) в результате ударных взаимодействий все фазовые точки множества $\Omega_{0}\left(M^{*}\right)$ его покидают (в силу условия $\left.H_{11}\left(x_{1}^{-}, \ldots, x_{n-1}^{-}\right) \leqslant 0\right)$;

б) при уменьшении времени $t$ из точек множества $\Omega_{0}\left(M^{*}\right)$ выходят $[9$, с. 171] в область $x_{n}>0$ фазовые траектории системы (2).

Осталось лиш выбрать достаточно малое время движения по этим траекториям и обозначить через $\Omega$ область, занимаемую этими траекториями.

В силу изложенного вьше вторая часть леммы следует из доказательства теоремы 10 [2, с. 40-41] о выпрямляемых семействах. Лемма доказана.

ЛЕмма 2. Пусть в точке $M^{*}$ выполняются условия (6). Если отобрахсение (1) имеет однозначное обратное отображение для любой точки $\left(\bar{x}_{1} \geqslant 0, \bar{x}_{2}, \ldots, \bar{x}_{n-1}\right)$, mo

1) существует достаточно малая окрестность $\Omega$ точки $M^{*}$ в фазовом пространстве системы (1), (2) такая, что в точки множества $\Omega_{0}=\Omega \cap$ $\left\{\left(x_{1}, \ldots, x_{n}\right) \mid x_{n}=0\right\}$ фазовые точки попадают (в силу ударных взаимодействий) из точек множества $\dot{x}_{n}<0, x_{n}=0$, а из точек множества $\Omega_{0}$ выходят в область $x_{n}>0$ фазовые траектории системы (2), составляющие все мнохество $\Omega$;

2) для любых систем вида (1), (2) (при одном и том жее значении п) локальные особенности вида (6) имеют достаточно малье окрестности в фазовом пространстве соответствующих систем, топологическая структура которых одинакова.

На рис. 1 особенности этого типа соответствует, например, точка $\mathrm{N}_{2}$.

ДокАЗАТЕЛЬСтво. На гиперповерхности удара $x_{n}=0$ можно выбрать достаточно малую окрестность $\Omega_{0}\left(M^{*}\right)$ точки $M^{*}$ такую, что во всех точках окрестности вьполняются условия (6), а значит, и неравенство $x_{1}>0$ (в силу (4) и (3)). Тогда

а) в силу существования обратного отображения для (1) и неравенства $H_{11} \leqslant 0$, в точки окрестности $\Omega_{0}\left(M^{*}\right)$ фазовые точки попадают (в результате ударных взаимодействий) из точек $\left(x_{1}^{-}, x_{2}^{-}, \ldots, x_{n-1}^{-}, 0\right)$, где $\dot{x}_{n}<0$;

б) при увеличении времени $t$ из точек множества $\Omega_{0}\left(M^{*}\right)$ выходят $[9$, c. 171] в область $x_{n}>0$ фазовые траектории системы дифференциальных уравнений (2). 
Осталось выбрать достаточно малое время движения по этим траекториям и обозначить через $\Omega$ область, занимаемую этими траекториями.

Справедливость второй части леммы 2 следует из доказательства теоремы 10 [2, c. 40-41] о вьпрямляемых семействах. Лемма доказана.

Лемма 3. Пусть в точке $M^{*}$ выполняются условия (7). Тогда существует такая достаточно малая окрестность $\Omega$ точки $M^{*}$ в фазовом пространстве системы (1), (2), которая множеством траекторий системы (2), выходяших при увеличении и уменьшении $t$ из точек множества $x_{n}=0, \dot{x}_{n}=\Phi_{n}\left(x_{1}, \ldots, x_{n}\right)=0$, разбивается на две части: фазовые траектории из первой части проходят в области $\Omega$, не пересекая гиперповерхность удара, а фазовые траектории из второй части представляют собой участки фазовых траекторий системы (2), которые приводят фазовые точки на гиперповерхность удара при $\dot{x}_{n}<0, x_{n}=0$, затем в силу ударных взаимодействий (1) фазовые точки переходят в точки множества $x_{n}=0, \dot{x}_{n} \geqslant 0$, после чего фазовые точки выходят в область $x_{n}>0$ по траекториям системы (2).

Любые локальные особенности $M^{*}\left(0, x_{2}^{*}, \ldots, x_{n-1}^{*}, 0\right)$ вида (7) систем (1), (2) (при одном и том жее значении $n$ и $\left.H_{11}\left(0, x_{2}^{*}, \ldots, x_{n-1}^{*}\right)<0\right)$ имеют достаточно малые окрестности в фазовом пространстве соответствующих систем, топологическая структура которых одинакова.

На рис. 1 особенности такого типа соответствует, например, точка $N_{3}$.

ДокАЗАТЕЛЬСТво. На гиперповерхности удара $x_{n}=0$ можно выбрать малую окрестность $\widetilde{\Omega}=\widetilde{\Omega}\left(M^{*}\right)$ изучаемой точки $M^{*}$ такую, что

1) множество $\widetilde{\Omega}$ замкнуто;

2 в в точках множества $\Omega_{0}^{-}\left(M^{*}\right)=\widetilde{\Omega} \cap\left\{\left(x_{1}, \ldots, x_{n}\right) \mid x_{n}=0, x_{1}<0\right\}$ справедливо неравенство $\dot{x}_{n}<0$, а в точках множества $\Omega_{0}^{0}\left(M^{*}\right)=\widetilde{\Omega} \cap\left\{\left(x_{1}, \ldots, x_{n}\right) \mid x_{n}=0\right.$, $\left.x_{1}=0\right\}$ вьполняются условия $\dot{x}_{n}=0, \ddot{x}_{n}>0$.

В силу ударных взаимодействий $(1)$ фазовые точки множества $\Omega_{0}^{-}$переходят в точки некоторого множества $\Omega_{0}^{+}$, где вьполнены условия (6) или (7).

Пусть замкнутое множество $\Omega_{1}\left(M^{*}\right)$ - часть гиперплоскости $x_{1}=0$, лежащая в достаточно малой окрестности точки $M^{*}$ в фазовом пространстве системы $(1),(2)$, такая, что

1) $\Omega_{0}^{0}=\Omega_{1} \cap\left\{\left(x_{1}, \ldots, x_{n}\right) \mid x_{n}=0\right\}$;

2 ) в точках множества $\Omega_{1}$ имеет место неравенство $\dot{x}_{1}>0$, которое при $x_{1}=0$, $x_{n}=0$ эквивалентно выполнению неравенства $\ddot{x}_{n}>0$, так как в силу $(2)$

$$
\left.\ddot{x}_{n}\right|_{x_{1}=0, x_{n}=0}=\left.\left.\dot{x}_{1}\right|_{x_{1}=0, x_{n}=0} \cdot \Phi_{n 1}\right|_{x_{1}=0, x_{n}=0} .
$$

Согласно [9, с. 171] из точек множества $\Omega_{0}^{-}$при уменьшении времени $t$, а из точек множества $\Omega_{0}^{+}$при увеличении $t$ выходят в область $x_{n}>0$ фазовые траектории системы (2); из точек множества $\Omega_{0}^{0}$ выходят фазовые траектории системы $(2)$, которые касаются гиперповерхности $x_{n}=0$ и выходят в область $x_{n}>0$ как при уменьшении, так и при увеличении $t$; из точек множества $\Omega_{1}$ выходят фазовые траектории системы $(2)$, при увеличении $t$ уходяшие в область $x_{1}>0$, а при уменьшении $t$ - в область $x_{1}<0$, пересечь гиперповерхность удара $x_{n}=0$, находясь в малой окрестности точки $M^{*}$, эти траектории не могут (в силу единственности решений системы (2)). 
Выбирая достаточно малое время $\Theta$ движения как в положительном, так и в отрицательном направлениях по указанным траекториям системы (2), выходящим из точек множеств $\Omega_{0}^{-}, \Omega_{0}^{0}, \Omega_{0}^{+}, \Omega_{1}$, обозначая через $\Omega\left(M^{*}, \Theta\right)$ область, занимаемую этими траекториями, можно завершить доказательство первой части леммы.

Для доказательства второй части леммы (о топологической эквивалентности) можно рассмотреть две точки $M^{*}$ и $N^{*}$ каких-либо двух систем вида $(1),(2)$, координаты которых удовлетворяют условиям (7), и построить, как выше, окрестности $\Omega\left(M^{*}, \Theta\right)$ и $\Omega\left(N^{*}, \Theta_{1}\right)$. Следуя [4, с. 203], сначала можно построить

1) топологическое (т.е. взаимно однозначное и взаимно непрерывное) отображение $N=\varphi(M)$ объединения $\Omega_{0}^{-}\left(M^{*}\right) \cup \Omega_{0}^{0}\left(M^{*}\right)$ на множество $\Omega_{0}^{-}\left(N^{*}\right) \cup \Omega_{0}^{0}\left(N^{*}\right)$, так чтобы $N^{*}=\varphi\left(M^{*}\right)$;

$2)$ продолжить отображение $\varphi$ как топологическое на множество $\Omega_{1}\left(M^{*}\right)$, так чтобы $\varphi\left(\Omega_{1}\left(M^{*}\right)\right)=\Omega_{1}\left(N^{*}\right)$.

Затем, обозначая через $G_{M} *\left(\right.$ соответственно $\left.G_{N^{*}}\right)$ отображение (1) для системы, имеющей особенность $M^{*}$ (соответственно $\left.N^{*}\right)$, можно доопределить отображение $\varphi$ на множестве $\Omega_{0}^{+}\left(M^{*}\right)$ следующим образом: если $M \in \Omega_{0}^{+}\left(M^{*}\right)$, то $\varphi(M)=N=$ $G_{N^{*}}\left(\varphi\left(G_{M^{*}}^{-1}(M)\right)\right) \in \Omega_{0}^{+}\left(N^{*}\right)$. Так доопределенное отображение $\varphi$ является топологическим на множестве $\Omega_{0}^{+}\left(M^{*}\right)$, поскольку таковыми на соответствующих множествах являются отображения $\varphi, G_{M^{*}}^{-1}, G_{N^{*}}$. Отображение $G_{M^{*}}\left(\right.$ соответственно $\left.G_{N^{*}}\right)$ является топологическим, так как в силу условия $H_{11}\left(0, x_{2}^{*}, \ldots, x_{n-1}^{*}\right) \neq 0$ оно имеет однозначное и гладкое обратное отображение по теореме о неявной функции [10, с. 294].

Теперь можно доопределить отображение $\varphi$ во всех точках окрестностей $\Omega\left(M^{*}, \Theta\right)$ и $\Omega\left(N^{*}, \Theta_{1}\right)$, сопоставив фазовые траектории соответствующих систем $(2)$ :

$$
b=\varphi(a), \quad a=\psi_{M^{*}}(t, M) \in \Omega\left(M^{*}, \Theta\right), \quad b=\psi_{N^{*}}\left(\Theta_{1} \Theta^{-1} t, N\right) \in \Omega\left(N^{*}, \Theta_{1}\right),
$$

где $\psi_{M^{*}}(t, M)$ и $\psi_{N^{*}}(t, N)$ - общее решение соответствующих систем $(2)$ с начальньми условиями $M=\psi_{M^{*}}(t=0, M)$ и $N=\psi_{N^{*}}(t=0, N) ; N=\varphi(M)$, а точки $M$ и $N$ принадлежат соответственно множествам $\Omega_{0}^{-}\left(M^{*}\right)$ и $\Omega_{0}^{-}\left(N^{*}\right), \Omega_{0}^{0}\left(M^{*}\right)$ и $\Omega_{0}^{0}\left(N^{*}\right), \Omega_{0}^{+}\left(M^{*}\right)$ и $\Omega_{0}^{+}\left(N^{*}\right), \Omega_{1}\left(M^{*}\right)$ и $\Omega_{1}\left(N^{*}\right)$; переменная $t$ принимает значения из интервала $[-\Theta, 0]$, если $M \in \Omega_{0}^{-}\left(M^{*}\right)$, из интервала $[0, \Theta]$, если $M \in \Omega_{0}^{+}\left(M^{*}\right)$, из интервала $[-\Theta, \Theta]$, если $M \in \Omega_{1}\left(M^{*}\right), \Omega_{0}^{0}\left(M^{*}\right)$.

Отображение $\varphi$ - гомеоморфизм в силу единственности и непрерьвной зависимости от начальных условий решений систем дифференциальных уравнений. Кроме того, $\varphi$ переводит фазовые траектории окрестности $\Omega\left(M^{*}, \Theta\right)$ в фазовые траектории окрестности $\Omega\left(N^{*}, \Theta_{1}\right)$. Лемма доказана.

4. Изучение локальной особенности типа 4). На рис. 1 особенности типа 4) соответствует, например, точка $M^{*}$.

В силу (4) равенство $\dot{x}_{n}=0$ при выполнении $x_{n}=0$ эквивалентно равенству $x_{1}=0$, поэтому изучение поведения фазовых траекторий системы $(1),(2)$ в окрестности точки $M^{*}$ может быть проведено с помошь следуюшего отображения $T$ многообразия $x_{n}=0, x_{1} \geqslant 0$ в себя [7]: $T=T_{2} T_{1}$, где отображение $T_{1}$ множества $x_{1} \geqslant 0, x_{n}=0$ на множество $x_{1} \leqslant 0, x_{n}=0$ осуществляется траекториями системы $(2)$, а отображение $T_{2}$ множества $x_{1} \leqslant 0, x_{n}=0$ на множество $x_{1} \geqslant 0, x_{n}=0$ происходит в соответствии с формулами (1) ударных взаимодействий. 
Отображение $T_{1}$, а значит и отображение $T$, заведомо существует в соответствующей части достаточно малой окрестности точек многообразия $Г$. Здесь через Г обозначается многообразие локальных особенностей типа 4).

В достаточно малой окрестности $\Omega$ точки $M^{*}=\left(x_{1}^{*}=0, x_{2}^{*}, \ldots, x_{n-1}^{*}, x_{n}^{*}=0\right)$ на многообразии $x_{n}=0, x_{1} \geqslant 0$ отображение $T$ можно записать [7] в виде

$$
\begin{gathered}
\bar{x}_{1}=R x_{1}+x_{1} \varphi_{1}\left(x_{1}, \ldots, x_{n-1}\right)=g_{1} \\
\bar{x}_{i}=x_{i}+x_{1}\left(c_{i}+\varphi_{i}\left(x_{1}, \ldots, x_{n-1}\right)\right)=g_{i}, \quad i=2, \ldots, n-1,
\end{gathered}
$$

где

$$
\begin{gathered}
R=-H_{11}\left(0, x_{2}^{*}, \ldots, x_{n-1}^{*}\right), \\
c_{i}=-b_{i}-2 a_{i} a_{1}^{-1}, \quad b_{i}=H_{1 i}\left(0, x_{2}^{*}, \ldots, x_{n-1}^{*}\right), a_{j}=\Phi_{j}\left(0, x_{2}^{*}, \ldots, x_{n-1}^{*}, 0\right), \\
\varphi_{j}\left(0, x_{2}^{*}, \ldots, x_{n-1}^{*}\right)=0, \quad \varphi_{j} \in C^{m-1}\left(\bar{\Omega}\left(M^{*}\right)\right), \quad j=1, \ldots, n-1 .
\end{gathered}
$$

В силу $(3),(8)$ и $(9)$ в точке $M^{*}$ выполняется $\ddot{x}_{n}=\Phi_{1} \Phi_{n 1}<0$ и $a_{1}<0$.

Кроме того, справедливы [7] следующие лемма и теорема.

Лемма 4. Для любой точки $M^{*}$ многообразия $Г$ можнно указать при $0<R<1$ такую достаточно малую окрестность на многообразии $x_{n}=0$, из кажсдой точки которой при $x_{1}>0$ выходит фазовая траектория, соответствующая бесконечноударному движению. Это движение оканчивается в некоторой точке многообразия Г.

Под бесконечноударным движением, здесь и далее, понимается движение с бесконечньм числом ударных взаимодействий за конечное время [11]. Траектория бесконечноударного движения, выходящая при $x_{n}=0$ из точки $M\left(x_{1}>0, x_{2}, \ldots, x_{n-1}\right)$ и оканчивающаяся в точке $M_{\infty}$, оставляет на многообразии $x_{n}=0$ “след" (рис. 1) в виде бесконечного числа точек

$$
M_{j}=T^{j}(M), \quad j=1,2, \ldots
$$

Теорема 1. Пусть величина $R, 0 \leqslant R<1$, определена формулами (10) $и M^{*}=$ $\left(0, x_{2}^{*}, \ldots, x_{n-1}^{*}, 0\right) \in \Gamma$. Тогда для любой точки $M$ из достаточно малой окрестности $M^{*}$ на многообразии $x_{1}>0, x_{n}=0$ все точки (11) лежат на проходящей через $M$ интегральной кривой системы дифференциальных уравнений

$$
\frac{d x_{i}}{d x_{1}}=f_{i}\left(x_{1}, \ldots, x_{n-1}\right), \quad i=2, \ldots, n-1,
$$

әде $f_{i}$ - определенные при $x_{1} \geqslant 0$ функиии класса $C^{m-2}, f_{i}=c_{i}(R-1)^{-1}+\widetilde{f}_{i}$, $\tilde{f}_{i}\left(0, x_{2}^{*}, \ldots, x_{n-1}^{*}\right)=0, i=2, \ldots, n-1$.

ТЕОРема 2. Для любых систем вида (1), (2) (при одном и том жсе значении $n$ ) локальные особенности $M^{*}\left(0, x_{2}^{*}, \ldots, x_{n-1}^{*}, 0\right)$ типа 4) имеют достаточно малье окрестности в фазовом пространстве соответствующих систем, топологическая структура которых одинакова, если только в точке $M^{*}$ имеет место

$$
-1<H_{11}\left(0, x_{2}^{*}, \ldots, x_{n-1}^{*}\right)<0 .
$$


ДокАЗАТЕЛЬство. Пусть наряду с (1), (2) задана система того же вида, т.е. определяемая при $y_{n}=0$ соотношениями

$$
\begin{gathered}
\bar{y}_{1}=y_{1}^{-} \widetilde{H}_{11}\left(y_{1}^{-}, \ldots, y_{n-1}^{-}\right)=\widetilde{H}_{1} \\
\bar{y}_{i}=y_{i}^{-}+y_{1}^{-} \widetilde{H}_{1 i}\left(y_{1}^{-}, \ldots, y_{n-1}^{-}\right)=\widetilde{H}_{i}, \quad i=2, \ldots, n-1,
\end{gathered}
$$

а при $y_{n}>0$ уравнениями

$$
\begin{gathered}
\dot{y}_{i}=\widetilde{\Phi}_{i}\left(y_{1}, \ldots, y_{n}\right), \quad i=1, \ldots, n-1 \\
\dot{y}_{n}=\widetilde{\Phi}_{n}\left(y_{1}, \ldots, y_{n}\right)=\widetilde{\Phi}_{n 1}\left(y_{1}, \ldots, y_{n}\right) y_{1}+y_{n} \widetilde{\Phi}_{n n}\left(y_{1}, \ldots, y_{n}\right),
\end{gathered}
$$

где соответствующие функции удовлетворяют условиям п. 1 . Пусть $M^{*}=\left(x_{1}^{*}=0, x_{2}^{*}\right.$, $\left.\ldots, x_{n-1}^{*}, x_{n}^{*}=0\right)$ и $N^{*}=\left(0, y_{2}^{*}, \ldots, y_{n-1}^{*}, 0\right)$ - любые две точки типа 4$)$ систем $(1),(2)$ и $(13),(14)$ соответственно.

В силу теоремы 1 точки $M^{*}$ и $N^{*}$ имеют такие малые окрестности $D_{r_{1}}=D_{r_{1}}\left(M^{*}\right)$ и $D_{r_{2}}=D_{r_{2}}\left(N^{*}\right)$ соответственно на многообразиях $x_{n}=0, x_{1} \geqslant 0$ и $y_{n}=0, y_{1} \geqslant 0$, где $r_{1}>0$ и $r_{2}>0$ обозначают наибольший диаметр соответствуюших окрестностей, в которых бесконечноударные движения системы (1), (2) описьваются уравнениями (12), а системы (13), (14) - уравнениями

$$
\frac{d y_{i}}{d y_{1}}=\widetilde{f}_{i}\left(y_{1}, \ldots, y_{n-1}\right), \quad i=2, \ldots, n-1 .
$$

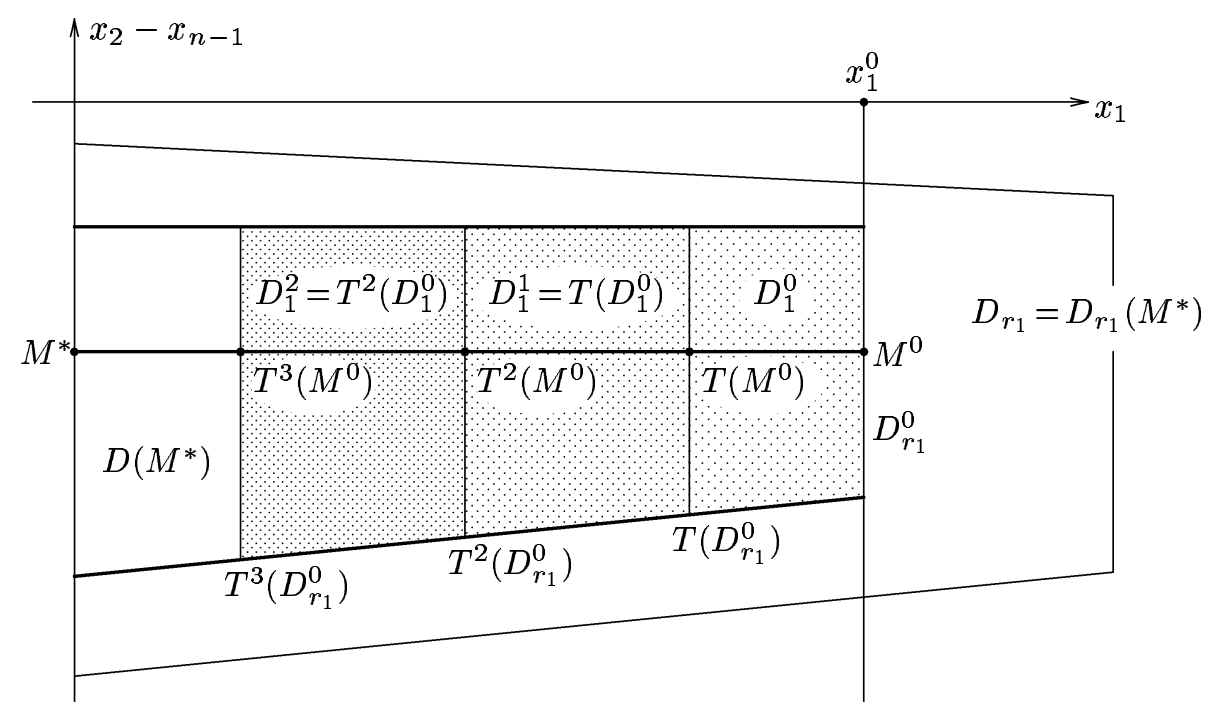

Рис. 2

Поскольку эти дифференциальные уравнения являются гладкими, можно выбрать определяемые ниже величины $x_{1}^{0}$ и $y_{1}^{0}$ настолько мальми, что (рис. 2)

1) из точек множеств $D_{r_{1}}^{0}$ и $D_{r_{2}}^{0}$, которые являются частями множеств $x_{1}=x_{1}^{0}>0$ и $y_{1}=y_{1}^{0}>0$ на гиперповерхностях $x_{n}=0$ и $y_{n}=0$, лежаших внутри множеств $D_{r_{1}}$ и $D_{r_{2}}$ и гомеоморфных шару размерности $n-2$, выходят фазовые траектории 
указанных дифференциальных уравнений, целиком лежащие при $0 \leqslant x_{1} \leqslant x_{1}^{0}$ и $0 \leqslant y_{1} \leqslant y_{1}^{0}$ в областях $D_{r_{1}}$ и $D_{r_{2}} ;$

2) траектория дифференциальных уравнений (12) либо (15), которая проходит через точку $M^{*}$ либо $N^{*}$, пересекает множество $D_{r_{1}}^{0}$ либо $D_{r_{2}}^{0}$ во внутренней точке $M^{0}$ либо $N^{0}$ этого множества;

3) точка $M^{0}$ либо $N^{0}$ при гомеоморфизме множества $D_{r_{1}}^{0}$ либо $D_{r_{2}}^{0}$ на шар размерности $n-2$ соответствует центру шара.

Пусть теперь $D\left(M^{*}\right)$ либо $D\left(N^{*}\right)$ есть область на гиперплоскости $x_{n}=0$ либо $y_{n}=0$, которую заполняют при $0 \leqslant x_{1} \leqslant x_{1}^{0}$ либо при $0 \leqslant y_{1} \leqslant y_{1}^{0}$ траектории дифференциальных уравнений $(12)$ либо $(15)$, проходящие через точки множества $D_{r_{1}}^{0}$ либо $D_{r_{2}}^{0}$ (на рис. 2 траектории дифференциальных уравнений (12) выделены жирными линиями).

Пусть $\widetilde{T}$ - отображение, определяемое соотношениями (10), но для системы $(13),(14)$, в точках множества $D_{r_{2}}\left(N^{*}\right)$. Точки образа $T\left(D_{r_{1}}^{0}\right)$ либо $\widetilde{T}\left(D_{r_{2}}^{0}\right)$ множества $D_{r_{1}}^{0}$ либо $D_{r_{2}}^{0}$ при отображении $T$ либо $\widetilde{T}$ принадлежат для достаточно малых $r_{1}$ либо $r_{2}$ пересечению множеств $D\left(M^{*}\right)$ и $x_{1}<x_{1}^{0}$ соответственно $D\left(N^{*}\right)$ и $y_{1}<y_{1}^{0}$, поскольку справедливо неравенство

$$
\bar{x}_{1} \leqslant(R+q) x_{1}, \quad q>0, \quad R+q<1,
$$

вытекающее из (10).

Пусть $D_{1}^{0}\left(M^{*}\right)$ - замкнутое множество, которое заполнено траекториями дифференциальных уравнений $(12)$, заключенными между множествами $T\left(D_{r_{1}}^{0}\right)$ и $D_{r_{1}}^{0}$. Соответственно, пусть $D_{2}^{0}\left(N^{*}\right)$ - замкнутое множество, которое заполнено траекториями дифференциальных уравнений $(15)$, заключенными между множествами $T\left(D_{r_{2}}^{0}\right)$ и $D_{r_{2}}^{0}$. Пусть $G$ - топологическое отображение множества $D_{r_{1}}^{0}$ на $D_{r_{2}}^{0}$, при котором $N^{0}=G\left(M^{0}\right)$ ( $G$ существует в силу выбора величин $x_{1}^{0}, y_{1}^{0}$ и множеств $\left.D_{r_{1}}^{0}, D_{r_{2}}^{0}\right) ; \varphi\left(x_{1}, M\right)$ - общее решение системы (12) с начальными условиями $M=\varphi\left(x_{1}^{0}, M\right) ; \psi\left(y_{1}, N\right)$ - общее решение системы (15) с начальными условиями $N=\psi\left(y_{1}^{0}, N\right)$. Тогда, определяя отображение $G$ на всем множестве $D_{1}^{0}\left(M^{*}\right)$ как

$$
G\left(\varphi\left(x_{1}, M\right)\right)=\psi\left(y_{1}^{0}-\left(x_{1}^{0}-x_{1}\right)\left(y_{1}^{0}-\widetilde{y}_{1}(N)\right)\left(x_{1}^{0}-\widetilde{x}_{1}(M)\right)^{-1}, N\right),
$$

где $0<\widetilde{x}_{1}(M) \leqslant x_{1} \leqslant x_{1}^{0}, 0<\widetilde{y}_{1}(N) \leqslant y_{1} \leqslant y_{1}^{0}, N=G(M), M \in D_{r_{1}}^{0}, N \in D_{r_{2}}^{0}$, $\varphi\left(\widetilde{x}_{1}(M), M\right) \in T\left(D_{r_{1}}^{0}\right), \psi\left(\widetilde{y}_{1}(N), N\right) \in \widetilde{T}\left(D_{r_{2}}^{0}\right)$, можно получить топологическое отображение $D_{1}^{0}\left(M^{*}\right)$ на $D_{2}^{0}\left(N^{*}\right)$ (в силу единственности и непрерьвной зависимости от начальных условий решения системы дифференциальных уравнений).

Теперь можно ввести на множестве $D\left(M^{*}\right)$ отображение $G_{T}$

$$
G_{T}\left(T^{p}(M)\right)=\widetilde{T}^{p}(N), \quad p=0,1, \ldots,
$$

где $M \in D_{1}^{0}\left(M^{*}\right), N \in D_{2}^{0}\left(N^{*}\right), N=G(M)$. Отображение $G_{T}$ обладает следующими свойствами.

Свойство 1. Отображение $G_{T}$ может быть определено для любой точки мнохсества $D\left(M^{*}\right)$ как переводящее все множество $D\left(M^{*}\right)$ на $D\left(N^{*}\right)$.

Действительно,

$$
T\left(D\left(M^{*}\right)\right)=\left(D\left(M^{*}\right) \backslash D_{1}^{0}\right) \cup T\left(D_{r_{1}}^{0}\right), \quad \widetilde{T}\left(D\left(N^{*}\right)\right)=\left(D\left(N^{*}\right) \backslash D_{2}^{0}\right) \cup \widetilde{T}\left(D_{r_{2}}^{0}\right),
$$


так как отображения $T$ при достаточно малом $r_{1}$ и $\widetilde{T}$ при достаточно малом $r_{2}$ взаимно однозначны и взаимно непрерьвны в силу (10); множества $x_{1}=0$ и $y_{1}=0$ при соответствующих отображениях $T$ и $\widetilde{T}$ переходят сами в себя; $T\left(D_{r_{1}}^{0}\right) \subset\left(D\left(M^{*}\right) \backslash D_{r_{1}}^{0}\right)$ и $\widetilde{T}\left(D_{r_{2}}^{0}\right) \subset\left(D\left(N^{*}\right) \backslash D_{r_{2}}^{0}\right)$. Поэтому $T^{2}\left(D_{r_{1}}^{0}\right) \subset\left(D\left(M^{*}\right) \backslash D_{1}^{0}\right)$ и $\widetilde{T}^{2}\left(D_{r_{2}}^{0}\right) \subset\left(D\left(N^{*}\right) \backslash D_{2}^{0}\right)$. Отсюда $T\left(D_{1}^{0}\right)=D_{1}^{1}$ и $\widetilde{T}\left(D_{2}^{0}\right)=D_{2}^{1}$ - замкнутые множества, заполненные траекториями дифференциальных уравнений (12) и (15) соответственно, заключенными между множествами $T\left(D_{r_{1}}^{0}\right)$ и $T^{2}\left(D_{r_{1}}^{0}\right)$ соответственно $\widetilde{T}\left(D_{r_{2}}^{0}\right)$ и $\widetilde{T}^{2}\left(D_{r_{2}}^{0}\right)$. При этом $D_{1}^{1} \cap D_{1}^{0}=$ $T\left(D_{r_{1}}^{0}\right)$ соответственно $D_{2}^{1} \cap D_{2}^{0}=\widetilde{T}\left(D_{r_{2}}^{0}\right)$ (рис. 2).

По индукции с помощью аналогичных рассуждений можно установить, что для любого целого $p>0 T^{p}\left(D_{1}^{0}\right)=D_{1}^{p}$ и $\widetilde{T}^{p}\left(D_{2}^{0}\right)=D_{2}^{p}$ - замкнутые множества, заполненные траекториями дифференциальных уравнений (12) и (15) соответственно, заключенными между множествами $T^{p}\left(D_{r_{1}}^{0}\right)$ и $T^{p+1}\left(D_{r_{1}}^{0}\right)$ соответственно $\widetilde{T}^{p}\left(D_{r_{2}}^{0}\right)$ и $\widetilde{T}^{p+1}\left(D_{r_{2}}^{0}\right)$. При этом

$$
\begin{array}{ll}
T^{p+1}\left(D_{r_{1}}^{0}\right) \subset\left(D\left(M^{*}\right) \backslash \bigcup_{k=0}^{p-1} D_{1}^{k}\right), & D_{1}^{p} \cap D_{1}^{p-1}=T^{p}\left(D_{r_{1}}^{0}\right), \\
\widetilde{T}^{p+1}\left(D_{r_{2}}^{0}\right) \subset\left(D\left(N^{*}\right) \backslash \bigcup_{k=0}^{p-1} D_{2}^{k}\right), & D_{2}^{p} \cap D_{2}^{p-1}=\widetilde{T}^{p}\left(D_{r_{2}}^{0}\right) .
\end{array}
$$

Отсюда в силу (16) для любого сколь угодно малого $\varepsilon>0$ существует $k$ такое, что при всяком целом $p \geqslant k$ любая точка $P \in D_{1}^{p}$ принадлежит множеству $\left\{\left(x_{1}, \ldots, x_{n-1}\right) \mid\right.$ $\left.0<x_{1} \leqslant \varepsilon\right\} \cap D\left(M^{*}\right)$, а любая точка $\widetilde{P} \in D_{2}^{p}$ принадлежит множеству $\left\{\left(y_{1}, \ldots, y_{n-1}\right) \mid\right.$ $\left.0<y_{1} \leqslant \varepsilon\right\} \cap D\left(N^{*}\right)$.

Поэтому

а) для любой точки $M \in D\left(M^{*}\right) \cap\left\{\left(x_{1}, \ldots, x_{n-1}\right) \mid x_{1}>0\right\}$ либо $N \in D\left(N^{*}\right) \cap$ $\left\{\left(y_{1}, \ldots, y_{n-1}\right) \mid y_{1}>0\right\}$ и $M \notin T^{k}\left(D_{r_{1}}^{0}\right)$ либо $N \notin \widetilde{T}^{k}\left(D_{r_{2}}^{0}\right)$ при всех $k=0,1, \ldots$, существуют единственные целое $p \geqslant 0$ и точка $M_{p} \in D_{1}^{0}$ либо $N_{p} \in D_{2}^{0}$ такие, что $M_{p}=T^{-p}(M)$ либо $N_{p}=\widetilde{T}^{-p}(N)$;

б) если же при каком-то целом $k \geqslant 0 M \in T^{k}\left(D_{r_{1}}^{0}\right)$ либо $N \in \widetilde{T}^{k}\left(D_{r_{2}}^{0}\right)$, то существует единственная точка $M_{k} \in D_{r_{1}}^{0}$ либо $N_{k} \in D_{r_{2}}^{0}$ такая, что $M_{k}=T^{-k}(M)$ либо $N_{k}=\widetilde{T}^{-k}(N)$.

Итак, для любой точки $M \in D\left(M^{*}\right) \cap\left\{\left(x_{1}, \ldots, x_{n-1}\right) \mid x_{1}>0\right\}$ определено отображение $G_{T}$, которое имеет вид

$$
G_{T}(M)=\widetilde{T}^{p} G T^{-p}(M)
$$

где $p$ - целое число $(p \geqslant 0)$ такое, что $T^{-p}(M) \in D_{1}^{0} \backslash T\left(D_{r_{1}}^{0}\right)$.

Если $M \in D\left(M^{*}\right) \cap\left\{\left(x_{1}, \ldots, x_{n-1}\right) \mid x_{1}=0\right\}$, то существует и единственна по построению множества $D\left(M^{*}\right)$ точка $M_{\infty}^{0} \in D_{r_{1}}^{0}$ такая, что $\lim _{p \rightarrow+\infty} T^{p}\left(M_{\infty}^{0}\right)=M$, и можно положить

$$
G_{T}(M)=\lim _{k \rightarrow+\infty} \widetilde{T}^{k}\left(G\left(M_{\infty}^{0}\right)\right)
$$

где точка $N_{\infty}^{0}=G\left(M_{\infty}^{0}\right) \in D_{r_{2}}^{0}$, а предел в $(18)$ существует в силу построения множества $D\left(N^{*}\right)$.

Тем самым, из (17) и (18) следует справедливость свойства 1 и следующего свойства. 
Свойство 2. Отображсние $G_{T}$ переводит траектории $M, T(M), T^{2}(M), \ldots$, $M \in D\left(M^{*}\right)$, отображсения $T$ в траектории $N, \widetilde{T}(N), \widetilde{T}^{2}(N), \ldots, N \in D\left(N^{*}\right)$, отобрахсени $\widetilde{T}\left(\right.$ при $\left.N=G_{T}(M)\right)$.

Свойство 3. Отобрахсение $G_{T}$ взаимно однозначно (по определению) и является взаимно непрерывным.

Действительно, если $M \in D\left(M^{*}\right) \cap\left\{\left(x_{1}, \ldots, x_{n-1}\right) \mid x_{1}>0\right\}$ и $M \notin T^{k}\left(D_{r_{1}}^{0}\right)$ при $k=0,1, \ldots$, то $G_{T}$ взаимно непрерьвно в точке $M$ в силу (17).

Если $M \in T^{k}\left(D_{r_{1}}^{0}\right)$ при некотором целом $k>0$, то достаточно малая окрестность $\Omega(M)$ точки $M$ имеет вид $\Omega=\Omega(M)=\Omega_{1} \cup \Omega_{2}$, где $\Omega_{1}=\Omega \cap D_{1}^{k-1}, \Omega_{2}=\Omega \cap D_{1}^{k}$, $\Omega_{1} \cap \Omega_{2}=\Omega_{12} \subset T^{k}\left(D_{r_{1}}^{0}\right)$. Согласно (17) $G_{T}(\Omega)=\widetilde{T}^{k-1} G T^{1-k}\left(\Omega_{1}\right) \cup \widetilde{T}^{k} G T^{-k}\left(\Omega_{2}\right)$, при этом $\widetilde{T}^{k-1} G T^{1-k}\left(\Omega_{1}\right) \cap \widetilde{T}^{k} G T^{-k}\left(\Omega_{2}\right)=\widetilde{T}^{k} G T^{-k}\left(\Omega_{12}\right)=\widetilde{\Omega}_{12} \subset \widetilde{T}^{k}\left(D_{r_{2}}^{0}\right)$ и $N=$ $G_{T}(M) \in \widetilde{\Omega}_{12}$. Поэтому в силу гомеоморфности отображений $T, \widetilde{T}, G$ достаточно малая окрестность $\Omega(M)$ перейдет при отображении $G_{T}$ в любую заданную малую окрестность $\Omega(N)$ точки $N$. Аналогичные рассуждения доказьвают непрерывность $G_{T}^{-1}$ в точке $N$.

Если $M \in T^{0}\left(D_{r_{1}}^{0}\right)=D_{r_{1}}^{0}$, то свойство 3 справедливо, так как при $M \in D_{1}^{0}$ отображения $G$ и $G_{T}$ совпадают.

Если $M \in D\left(M^{*}\right) \cap\left\{\left(x_{1}, \ldots, x_{n-1}\right) \mid x_{1}=0\right\}$, то ее достаточно малая окрестность $\Omega(M)$ в области определения $D\left(M^{*}\right)$ отображения $G_{T}$ содержится во множестве $\Omega_{p}=D\left(M^{*}\right) \backslash \bigcup_{k=0}^{p} D_{1}^{k}$ при достаточно большом числе $p>0$ (см. обоснование свойства 1 ). Кроме того, все точки множества $D_{1}^{0}$, которые при итерациях отображения $T$ переходят в точки множества $\Omega(M)$, находятся на траекториях дифференциальных уравнений (12), лежащих (в силу непрерьвной зависимости решений дифференциальных уравнений от начальных условий) в малой окрестности траектории (12), соединяющей точку $M$ и соответствующую точку $M_{\infty}^{0} \in D_{r_{1}}^{0}$. Поэтому можно всегда найти достаточно большое $p>0$ и достаточно малую окрестность $\Omega(M) \subset \Omega_{p}$ такие, что в силу (16) $G_{T}(\Omega(M)) \subset D\left(N^{*}\right) \cap\left\{\left(y_{1}, \ldots, y_{n-1}\right) \mid y_{1}<\varepsilon\right\}$, где $\varepsilon$ - любое сколь угодно малое число $(\varepsilon>0)$, и множество $G_{T}(\Omega)$ лежит в сколь угодно малой окрестности фазовой траектории дифференциальных уравнений (15), проходящей через точки $N=G_{T}(M)$ и $N_{\infty}^{0}=G_{T}\left(M_{\infty}^{0}\right) \in D_{r_{2}}^{0}$.

Последнее означает, что отображение $G_{T}$ является непрерывным в точке $M \in D\left(M^{*}\right)$ $\cap\left\{\left(x_{1}, \ldots, x_{n-1}\right) \mid x_{1}=0\right\}$. Аналогично доказьвается непрерьвность обратного отображения $G_{T}^{-1}$ в точке $N=G_{T}(M) \in D\left(N^{*}\right) \cap\left\{\left(y_{1}, \ldots, y_{n-1}\right) \mid y_{1}=0\right\}$.

Справедливость свойств 1-3 отображения $G_{T}$ доказывает топологическую эквивалентность окрестностей $D\left(M^{*}\right)$ и $D\left(N^{*}\right)$ при действии отображений $T$ и $\widetilde{T}$. При этом $G_{T}\left(M^{*}\right)=N^{*}$ по определению отображения $G_{T}$.

Пусть $E\left(M^{*}\right)$ - окрестность точки $M^{*}$ в фазовом пространстве системы $(1),(2)$, которую заполняют участки траекторий системы (2), выходящие из точек множества $D\left(M^{*}\right)$ и пересекающие гиперповерхность удара $x_{n}=0 ; x_{i}=\varphi_{i}\left(t, x_{1}^{0}, x_{2}^{0}, \ldots, x_{n}^{0}\right)$ - общее решение системы (2) с начальньми условиями $x_{i}^{0}=\varphi_{i}\left(0, x_{1}^{0}, \ldots, x_{n}^{0}\right), i=1, \ldots, n$. Соответственно, пусть $E\left(N^{*}\right)$ - окрестность точки $N^{*}$ в фазовом пространстве системы (13), (14), которую заполняют участки траекторий системы (14), выходящие из точек множества $D\left(N^{*}\right)$ и пересекающие гиперповерхность удара $y_{n}=0 ; y_{i}=\psi_{i}\left(t, y_{1}^{0}\right.$, $\left.y_{2}^{0}, \ldots, y_{n}^{0}\right)$ - общее решение системы $(14)$ с начальными условиями $y_{i}^{0}=\psi_{i}\left(0, y_{1}^{0}, \ldots, y_{n}^{0}\right)$, $i=1, \ldots, n$. 
Тогда можно задать отображение $G_{0}$ окрестности $E\left(M^{*}\right)$ на $E\left(N^{*}\right)$ :

$$
G_{0}(M)=\left(\psi_{1}\left(|\tau| \widetilde{\theta} \theta^{-1}, y_{1}^{N}, \ldots, y_{n-1}^{N}, 0\right), \ldots, \psi_{n}\left(|\tau| \widetilde{\theta} \theta^{-1}, y_{1}^{N}, \ldots, y_{n-1}^{N}, 0\right)\right)
$$

где $M=\left(x_{1}, x_{2}, \ldots, x_{n-1}, x_{n}\right) \in E\left(M^{*}\right),\left(y_{1}^{N}, \ldots, y_{n-1}^{N}\right)=G_{T}\left(M_{-1}\right) ; M_{-1}=\left(\varphi_{1}(\tau\right.$, $\left.\left.x_{1}, \ldots, x_{n}\right), \ldots, \varphi_{n-1}\left(\tau, x_{1}, \ldots, x_{n}\right)\right)=\left(x_{-1}, x_{-2}, \ldots, x_{-(n-1)}\right) ; \tau-$ ближайший к 0 неположительньй корень уравнения $\varphi_{n}\left(t, x_{1}, \ldots, x_{n}\right)=0 ; \theta$ - ближайший к 0 положительный корень уравнения $\varphi_{n}\left(t, x_{-1}, x_{-2}, \ldots, x_{-(n-1)}, 0\right)=0 ; \widetilde{\theta}-$ ближайший к 0 положительньй корень уравнения $\psi_{n}\left(t, y_{1}^{N}, \ldots, y_{n-1}^{N}, 0\right)=0$.

В силу единственности и непрерывной зависимости решений системы дифференциальных уравнений от начальных условий и того, что $G_{T}$ - гомеоморфизм, величины $\tau, \theta, \widetilde{\theta}$ существуют, единственны и являются непрерывными функциями, причем $\tau \rightarrow 0$, $\theta \rightarrow 0, \widetilde{\theta} \rightarrow 0$ при $M=\left(x_{1}, x_{2}, \ldots, x_{n}\right) \rightarrow\left(0, p_{2}^{*}, \ldots, p_{n-1}^{*}, 0\right) \in E\left(M^{*}\right) \cap \Gamma$. Следовательно, отображение $G_{0}$, переводящее $E\left(M^{*}\right)$ в $E\left(N^{*}\right)$, является гомеоморфизмом, а по построению $G_{0}$ переводит траектории системы $(1),(2)$ в траектории системы $(13),(14)$. Последнее означает топологическую эквивалентность качественной структуры окрестностей $E\left(M^{*}\right)$ и $E\left(N^{*}\right)$. Теорема доказана.

\section{СПИСОК ЦИТИРОВАННОЙ ЛИТЕРАТУРЫ}

[1] Андронов А. А., Витт А. А., Хайкин С. Э. Теория колебаний. М.: Физматгиз, 1959.

[2] Немыцкий В. В., Степанов В. В. Качественная теория дифференциальных уравнений. 2-е изд. М.-Л.: ГИТТЛ, 1949.

[3] Андронов А. А., Леонтович Е. А., Гордон И. И., Майер А. Г. Качественная теория динамических систем второго порядка. М.: Наука, 1966.

[4] Филиппов А.Ф. Дифференциальные уравнения с разрьвной правой частью. М.: Наука, 1985.

[5] Денисов Г. Г., Неймарк Ю. И., Сандалов В. М., Цветков Ю. В. Об обкате ротора по жесткому подшипнику // Изв. АН СССР. Мех. твердого тела. 1973. № 4. С. 7-13.

[6] Федосенко Ю.С. О структуре фазового пространства и периодических движениях неавтономных динамических систем с ударными взаимодействиями // ПММ. 1976. Т. 40. № 4. C. $618-629$.

[7] Горбиков С. П., Неймарк Ю.И. Вспомогательные скользящие движения динамических систем с ударньми взаимодействиями // Дифференц. и интегральные уравнения. Межвузовский сб. Горький: ГГУ, 1981. С. 59-64.

[8] Горбиков С. П. Особенности строения фазового пространства динамических систем с ударными взаимодействиями // Изв. АН СССР. Мех. твердого тела. 1987. № 3. С. 23-26.

[9] Неймарк Ю. И. Методточечных отображений в теории нелинейных колебаний. М.: Наука, 1972.

[10] Шварц Л. Анализ. Т. 1. М.: Мир, 1972.

[11] Нагаев Р. Ф. Механические процессы с повторными затухающими соударениями. М.: Наука, 1985.

Научно-исследовательский институт прик ладной математики и кибернетики Нижегородского государственного университета им. Н. И. Лобачевского

Поступило 26.09 .95

Исправленный вариант 28.01 .98 